\title{
Adapting Petri Nets Reductions to Promela Specifications
}

\author{
C. Pajault ${ }^{1}$, J.-F. Pradat-Peyre ${ }^{1}$, and P. Rousseau ${ }^{2}$ \\ ${ }^{1}$ LIP6, Université Pierre et Marie Curie, Paris, \\ \{Christophe.Pajault, Jean-Francois.Pradat-Peyre\}@lip6.fr \\ 2 Cedric-CNAM \\ rousseau@cnam.fr
}

\begin{abstract}
The interleaving of concurrent processes actions leads to the well-known combinatorial explosion problem. Petri nets theory provides some structural reductions to tackle this phenomenon by agglomerating sequences of transitions into a single atomic transition. These reductions are easily checkable and preserve deadlocks, Petri nets liveness and any LTL formula that does not observe the modified transitions. Furthermore, they can be combined with other kinds of reductions such as partial-order techniques to improve the efficiency of state space reduction. We present in this paper an adaptation of these reductions for Promela specifications and propose simple rules to automatically infer atomic steps in the Promela model while preserving the checked property. We demonstrate on typical examples the efficiency of this approach and propose some perspectives of this work in the scope of software model checking.
\end{abstract}

\section{Introduction}

The interleaving of concurrent processes actions leads to a combinatory explosion. In order to give a simple insight of this problem, let us consider a simple example: let $\left\{p_{i}\right\}_{i=1 \ldots n}$ be a set of stateless servers which infinitely execute a loop

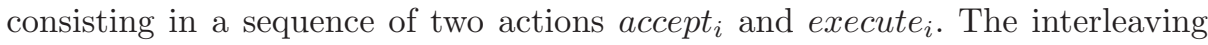
of these actions leads to a state space whose size is $2^{n}$. Partial order methods (e.g. persistent sets [1, sleep sets [2, stubborn sets [3, ...), or symmetry based reductions 45] may reduce the size of the state space to a size of $n$. However, the simple fact of considering the sequence as atomic leads to a state space reduced to a singleton! Obviously, as for partial order techniques, such a reduction may be faulty since for instance, it could hide occurrence of deadlocks. The goal of a reduction theory is to (syntactically) characterize situations where a reduction is sound and how to perform it.

Based on this principle, we proposed in [6] some new Petri nets reductions that cover a large range of synchronization patterns. We extended these reductions to colored Petri nets (which are an abbreviation of Petri nets) in [78] and use them in the QUASAR 9] platform that performs verification of concurrent Ada programs by analyzing an intermediate colored Petri net generated from a given program.

These reductions yield very interesting results and we present in this paper how these reductions can be adapted to simplify program analysis without needing a translation step into a more formal model (such as Petri nets). We illustrate 
our approach with the Promela language since it's a simple and clear language associated to the very efficient model checker Spin [10].

More precisely, we define some syntactical rules based on Petri nets agglomerations which allow the automatic detection of sequences of statements that can be marked as "atomic" (using the atomic construction of Promela) while preserving analyzed properties. The interest of this transformation is to significantly reduce interleaving and thus the size of the state space.

\section{Petri Nets Transitions Agglomerations}

A Petri net reduction is characterized by some application conditions, a net transformation and a set of preserved properties (i.e. which properties are simultaneously true or false in the original net and in the reduced one). Before presenting the pre- and the post-agglomerations, we briefly recall some Petri nets definitions.

\subsection{Brief Petri Nets Definitions and Notations}

Definition 1 (Petri net model). A marked net $\left(N, m_{0}\right)$ is defined by a tuple $\left(P, T, W^{-}, W^{+}, m_{0}\right)$ where: $P$ is the finite set of places, $T$ is the finite set of transitions disjoint from $P, W^{-}$(resp. $W^{+}$) an integer matrix indexed by $P \times T$ is the backward (resp. forward) incidence matrix, $m_{0}$ a integer vector indexed by $P$ is the initial marking. The transitions linked to a place $p$ are defined by • $p=\left\{t \mid W^{+}(p, t)>0\right\}$ and $p^{\bullet}=\left\{t \mid W^{-}(p, t)>0\right\}$.

Definition 2 (Firing rule). Let $\left(N, m_{0}\right)$ be a marked net then a transition $t \in T$ is firable from a marking $m$ (denoted by $m[t\rangle)$ iff $\forall p \in P m(p) \geq W^{-}(p, t)$. The firing of $t \in T$ firable from $m$ leads to the marking $m^{\prime}$ (denoted by $m[t\rangle m^{\prime}$ ) defined by $\forall p \in P m^{\prime}(p)=m(p)+W(p, t)$ where $W$ the incidence matrix is defined by $W=W^{+}-W^{-}$. A marking $m$ such that $\forall t \in T, N O T(m[t\rangle)$ is called a dead marking.

We use the following notations.

$-T^{*}$ is the set of finite sequences of transitions and $T^{\omega}$ is the set of infinite sequences of transitions; $\lambda$ defines the empty sequence of transitions;

$-\Pi_{T^{\prime}}(s)$ denotes the projection of the sequence $s$ on a subset of transitions $T^{\prime}$ and is recursively defined by $\Pi_{T^{\prime}}(\lambda)=\lambda, \forall t \in T^{\prime}, \Pi_{T^{\prime}}(s . t)=\Pi_{T^{\prime}}(s) . t$ and $\forall t \notin T^{\prime}, \Pi_{T^{\prime}}(s . t)=\Pi_{T^{\prime}}(s)$;

- $|s|_{T^{\prime}}=\left|\Pi_{T^{\prime}}(s)\right|$ denotes the number of occurrences of transitions of $T^{\prime}$ in $s$;

- Pref $(s)=\left\{s^{\prime} \mid \exists s^{\prime \prime}\right.$ s.t. $\left.s=s^{\prime} . s^{\prime \prime}\right\}$ denotes the set of prefixes of $s$.

Definition 3 (Firing rule extension). Let $\left(N, m_{0}\right)$ be a marked net. A finite sequence $s \in T^{*}$ is firable from $m$, a marking and leads to $m^{\prime}$ (also denoted by $m[s\rangle$ and $\left.m[s\rangle m^{\prime}\right)$ iff either $s=\lambda$ and $m^{\prime}=m$ or $s=s_{1}$.t with $t \in T$ and $\exists m_{1} m\left[s_{1}\right\rangle m_{1}$ and $m_{1}[t\rangle m^{\prime}$ We note Reach $\left(N, m_{0}\right)=\left\{m \mid \exists s \in T^{*} m_{0}[s\rangle m\right\}$ the set of reachable markings. An infinite sequence $s \in T^{\omega}$ is firable from $m a$ marking (also denoted $m[s\rangle$ ) iff for every finite prefix $s_{1}$ of $s, m\left[s_{1}\right\rangle$. 
Definition 4 (Generated language). Let $\left(N, m_{0}\right)$ be a marked net then

- $L\left(N, m_{0}\right)=\left\{s \in T^{*} \mid m_{0}[s\rangle\right\}$ is the language of finite sequences,

- $L^{\operatorname{Max}}\left(N, m_{0}\right)=\left\{s \in T^{*} \mid \exists m\right.$ dead marking $\left.m_{0}[s\rangle m\right\}$ is the language of finite maximal sequences,

- $L^{\omega}\left(N, m_{0}\right)=\left\{s \in T^{\omega} \mid m_{0}[s\rangle\right\}$ is the language of infinite sequences.

\subsection{Petri Nets Agglomerations}

We note $\left(N, m_{0}\right)$ a Petri net and we suppose in the following definitions that the set of transitions of the net is partitioned as : $T=T_{0} \biguplus_{i \in I} H_{i} \biguplus_{i \in I} F_{i}$ where $I$ denotes a non empty set of indices. The underlying idea of this decomposition is that a couple $\left(H_{i}, F_{i}\right)$ defines transitions sets that are causally dependent: an occurrence of $f \in F_{i}$ in a firing sequence may always be related to a previous occurrence of some $h \in H_{i}$ in this sequence. Starting from this property, we developed conditions on the behavior of the net which ensure that we can restrict the dynamics of the model to sequences where each occurrence $h \in H_{i}$ is immediately followed by an occurrence of some $f \in F_{i}$ without changing its behavior w.r.t. to a set of properties. This restricted behavior is the behavior of a reduced net, denoted $\left(N_{r}, m_{0}\right)$, defined in Appendix.

From now, we note $H=\cup_{i \in I} H_{i}$ and $F=\cup_{i \in I} F_{i}$. The firing rule in the reduced net is noted $\rangle_{r}$ (i.e. $m[s\rangle_{r} m^{\prime}$ denotes a firing sequence in the reduced net). We note also $\phi$ the homomorphism from the monoid $T_{r}^{*}$ to the monoid $T^{*}$ defined by: $\forall t \in T_{0}, \phi(t)=t$ and $\forall i \in I, \forall h \in H_{i}, \forall f \in F_{i}, \phi(h f)=h . f$ This homomorphism is extended to an homomorphism from $\mathcal{P}\left(T_{r}^{*}\right)$ to $\mathcal{P}\left(T^{*}\right)$ and from $\mathcal{P}\left(T_{r}^{\infty}\right)$ to $\mathcal{P}\left(T^{\infty}\right)$.

In order to obtain the preservation properties (such like deadlock occurrences) we have to introduce behavioral hypotheseses. The basic one, named Potential agglomerability ensures that an occurrence of a transition of $F$ is always preceeded by an occurrence of a transition of $H$. For doing that we define a set of counting functions, denoted $\Gamma_{i}$, by $\forall s \in T^{*}, \Gamma_{i}(s)=|s|_{H_{i}}-|s|_{F_{i}}$.

Definition 5 (P-agglomerability). A marked net $\left(N, m_{0}\right)$ is potentially agglomerable (p-agglomerable for short) iff $\forall s \in L\left(N, m_{0}\right), \forall i \in I, \Gamma_{i}(s) \geq 0$.

We define now the behavioral conditions that ensure that the agglomerations preserve properties of the net. Note that these behavioral conditions can be checked with efficient structural and algebraical sufficient conditions (not presented here) directly on the Petri net.

Pre-Agglomeration. The following definition states four conditions ensuring that delaying the firing of a transition $h \in H_{i}$ until some $f \in F_{i}$ fires does not modify the behavior of the net w.r.t. the set of properties we want to preserve.

Definition 6. Let $\left(N, m_{0}\right)$ be a p-agglomerable net. $\left(N, m_{0}\right)$ is

1. H-independent iff $\forall i \in I, \forall h \in H_{i}, \forall m \in \operatorname{Reach}\left(N, m_{0}\right), \forall s$ such that $\forall s^{\prime} \in \operatorname{Pref}(s), \Gamma_{i}\left(s^{\prime}\right) \geq 0, m[h . s\rangle \Longrightarrow m[s . h\rangle$

2. divergent-free iff $\forall s \in L^{\infty}\left(N, m_{0}\right),|s|_{T_{0} \cup F}=\infty$ 
3. quasi-persistent iff $\forall i \in I, \forall m \in R e a c h\left(N, m_{0}\right), \forall h \in H_{i}$, $\forall s \in\left(T_{0} \cup F\right)^{*}$, such that $m[h\rangle$ and $m[s\rangle \exists s^{\prime} \in\left(T_{0} \cup F\right)^{*}$ fulfilling: $m\left[h . s^{\prime}\right\rangle$, $\Pi_{F}\left(s^{\prime}\right)=\Pi_{F}(s)$ and $W\left(s^{\prime}\right) \geq W(s)$.

Furthermore, if $s \neq \lambda \Longrightarrow s^{\prime} \neq \lambda$ then the net is strongly quasi-persistent.

4. $H$-similar iff $|I|=1$ or $\forall i, j \in I, \forall m \in \operatorname{Reach}\left(N, m_{0}\right), \forall s \in T_{0}^{*}$, $\forall h_{i} \in H_{i}, \forall h_{j} \in H_{j}, \forall f_{j} \in F_{j} m\left[h_{i}\right\rangle$ and $m\left[s . h_{j} . f_{j}\right\rangle \Longrightarrow \exists s^{\prime} \in\left(T_{0}\right)^{*}, \exists f_{i} \in F_{i}$ such that $m\left[s^{\prime} . h_{i} . f_{i}\right\rangle$ and such that $s=\lambda \Longrightarrow s^{\prime}=\lambda$.

The $H$-independence roughly means that once a transition $h \in H_{i}$ is firable it can be delayed as long as one does not need its occurrence to fire a transition of $F_{i}$. When a net is divergent-free it does not generate infinite sequences with some suffix included in $H$. In the pre-agglomeration scheme, we transform original sequences by permutation and deletion of transitions to simulateable sequences. Such an infinite sequence cannot be transformed by this way into an infinite simulateable sequence. Therefore this condition is mandatory. The quasi-persistence ensures that in the original net a "quick" firing of a transition of $H$ does not lead to some deadlock which could have been avoided by delaying this firing. At last, the $H$-similarity forbids situations where the firing of transitions of $F$ is prevented due to a "bad" choice of a subset $H_{i}$.

Under previous conditions (or a subset of), fundamental properties of a net are preserved by the pre-agglomeration reduction. This result is stated in the following theorem whose demonstration is provided in [6].

Theorem 1. If a p-agglomerable Petri net $\left(N, m_{0}\right)$ is also

1. H-independent and divergent-free then

$$
\Pi_{T_{0} \cup F}\left(L^{\max }\left(N, m_{0}\right)\right) \supseteq \Pi_{T_{0} \cup F}\left(\Phi\left(L^{\max }\left(N_{r}, m_{0 r}\right)\right)\right)
$$

2. $H$-independent, strongly quasi-persistent and $H$-similar then

$$
\Pi_{T_{0} \cup F}\left(L^{\max }\left(N, m_{0}\right)\right) \subseteq \Pi_{T_{0} \cup F}\left(\Phi\left(L^{\max }\left(N_{r}, m_{0 r}\right)\right)\right)
$$

3. $H$-independent then

$$
\Pi_{T_{0} \cup F}\left(\phi\left(L^{\infty}\left(N_{r}, m_{0}\right)\right)\right)=\Pi_{T_{0} \cup F}\left(L^{\infty}\left(N, m_{0}\right)\right)
$$

The first point defines which conditions ensure that the reduction does not introduce maximal blocking sequences (e.g. characterizing a deadlock) in the reduced net. The second one fixes when the reduction does not hide some maximal blocking sequences. At last, the third point focuses on the preservation of properties expressed with infinite sequences (e.g. fairness properties).

Post-Agglomeration. The main behavioral property that the conditions of the post-agglomeration implies is the following one: in every firing sequence with an occurrence of a transition $h$ of $H$ followed later by an occurrence of a transition $f$ of $F$, one can immediately fire $f$ after $h$. From a modeling point of view, the set $F$ represents local actions while the set $H$ corresponds to global actions possibly involving synchronization. 
Definition 7. Let $\left(N, m_{0}\right)$ be a p-agglomerable marked net. $\left(N, m_{0}\right)$ is

1. F-independent iff $\forall i \in I, \forall h \in H_{i}, \forall f \in F_{i}, \forall s \in\left(T_{0} \cup H\right)^{*}, \forall m \in$ $\operatorname{Reach}\left(N, m_{0}\right), m[h . s . f\rangle \Longrightarrow m[h . f . s\rangle$

$\left(N, m_{0}\right)$ is strongly $F$-independent iff $\forall i \in I, \forall h \in H_{i}, \forall f \in F_{i}, \forall s \in T^{*}$ s.t. $\forall s^{\prime} \in \operatorname{Pref}(s), \Gamma\left(s^{\prime}\right) \geq 0 \forall m \in \operatorname{Reach}\left(N, m_{0}\right), m[$ h.s.f $\rangle \Longrightarrow m[$ h.f.s $\rangle$

2. F-continuable iff $\forall i \in I, \forall h \in H_{i}, \forall s \in T^{*}$, s.t. $\forall s^{\prime} \in \operatorname{Pref}(s), \Gamma\left(s^{\prime}\right) \geq 0$ $\forall m \in \operatorname{Reach}\left(N, m_{0}\right) m[h . s\rangle \Longrightarrow \exists f \in F_{i}$ such that $m[$ h.s.f $\rangle$

We express the strong dependence of the set $F$ on the set $H$ with these two hypotheses. The $F$-independence means that any firing of $f \in F$ may be anticipated just after the occurrence of a transition $h \in H$ which "makes possible" this firing. The $F$-continuation means that an excess of occurrences of $h \in H$ can always be reduced by subsequent firings of transitions of $F$.

As for the pre-agglomeration, these conditions (or a subset of) ensure that fundamental properties of a net are preserved by the post-agglomeration reduction (the demonstration is provided in [6]).

Theorem 2. If a p-agglomerable Petri net $\left(N, m_{0}\right)$ is also

1. F-continuable and F-independent then

$$
\Pi_{T_{0} \cup H}\left(L^{\max }\left(N, m_{0}\right)\right)=\Pi_{T_{0} \cup H}\left(\Phi\left(L^{\max }\left(N_{r}, m_{0 r}\right)\right)\right)
$$

2. F-continuable and strongly F-independent then

$$
\Pi_{T_{0} \cup H}\left(\phi\left(L^{\infty}\left(N_{r}, m_{0}\right)\right)\right)=\Pi_{T_{0} \cup H}\left(L^{\infty}\left(N, m_{0}\right)\right)
$$

\section{Simplifying Promela Model Analysis}

\subsection{The Promela Language}

Promela is a verification modeling language associated with the Spin tool. Promela specifications consist of processes, message channels and variables. Processes are global objects. Message channels and variables can be declared either globally or locally within a process. Processes specify behavior while channels and global variables define the processes environment.

The execution of every statement is conditional on its executability. Statements are either executable or blocked. For instance, an assignment $j=1$ is always executable while a boolean condition $j==1$ is executable only when $j$ is equal to 1 .

An important feature of Promela is the ability given to processes to synchronize themselves through message channels which are used to model the transfer of data from one process to another. They are declared either locally or globally.

Figure 1 depicts an example of Promela specification. This simple producer/consumer example spawns two processes: one of type writer and one of type reader. The writer sends $\mathrm{N}$ messages to the reader via a channel. 


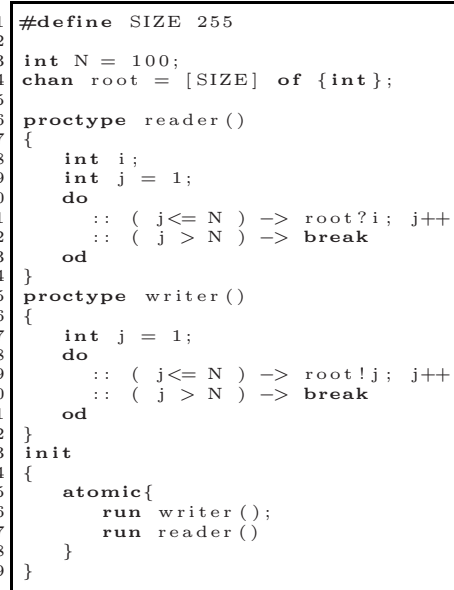

Fig. 1. A simple producer/consumer Promela model

On line 4 , chan root $=[$ SIZE $]$ of $\{$ int $\}$; declares a channel that can store up to SIZE messages of type int. The statement root! $j$ (line 19) is a transmission of the value of $j$ on the channel root (i.e. it appends the value to the tail of the channel). And root?i (line 11) models the reception on that channel (i.e. it retrieves it from the head of the channel, and stores it in the variable $i)$. The send operation is executable only when the channel addressed is not full. The receive operation, similarly, is only executable when the channel is non empty. The channels pass messages in a first-in-first-out order.

The control flow in Promela can be defined with the selection, the repetition, and the unconditional jumps. For instance, The lines 18-21 in Figure 1 contains a repetition statement (do ... od). This repetition statement contains two sequences of statements, each preceded by a double colon. The first statements of these execution sequences are called guards. This repetition sequence will either execute the sequence starting with : : $(j<=N)$ or the sequence starting with : : $(j>N)$ regarding which guard is executable. If several guards are executables, one is randomly chosen. Once the sequence is executed, the repetition statement will be repeated. The normal way to terminate the repetition structure is the use of the break statement. The selection statement is similar to the repetition statement, but occurs only once.

\subsection{Syntactical Promela Agglomerations}

We define now some conditions under which it is possible to automatically infer agglomerations in a Promela specification. These agglomerations group sequential statements into an atomic block in order to reduce the combinatory. In other term we will fix simple conditions that allow us to transform a sequence 1

\footnotetext{
${ }^{1}$ The case $k=0$ is obvious and not studied.
} 


$$
i_{0} ; \operatorname{atomic}\left\{i_{1} ; i_{2} ; \ldots ; i_{k}\right\}
$$

into the atomic sequence

$$
\text { atomic }\left\{i_{0} ; i_{1} ; i_{2} ; \ldots ; i_{k}\right\}
$$

We study different cases for which this transformation preserves deadlock, non progress cycles or any LTL formula that does not observe the action $i_{0}$ (when we perform a pre-agglomeration) or any actions of $i_{j}$ (when performing a post-agglomeration).

Methodology used The main principle is to explicitly use the behavioral conditions of Petri nets agglomerations for characterizing different cases in which the transformation is correct w.r.t. the analyzed property. Indeed, for any Promela specification, a corresponding Petri net with the same behavior can be generated (as in the QUASAR project for analyzing concurrent dynamic Ada programs) and adapted to Promela specification in [11. Then, agglomeration conditions on Petri nets can be translated into syntactical and semantical conditions in the Promela program.

In order to interpret Petri net behavior into Promela behavior we classify Promela statements following three criteria (in the following examples we suppose that a statement $i_{0}$ is followed by a sequence of statement $\left.s_{f}=\left\{i_{1} \ldots ; i_{k}\right\}\right)$ :

1. is the statement blocking or not? For instance, an assignment is a non blocking statement, because it is always executable while a boolean expression or a receive operation on a channel are blocking statements; this characteristic is related to the $F$-continuation hypothesis ;

Indeed, if the statement $i_{0}$ is followed by a sequence of non blocking statements $s_{f}$, we know that the sequence will always be executed if $i_{0}$ is executed and so the $F$-continuation hypothesis is fulfilled (in the Petri net model, all the transitions modeling the sequence execution will be executable after firing $\left.i_{0}\right)$.

2. does the statement refers local or global variables? When the statement refers only local variables, the value for which the statement is executed by a process cannot change by the execution of other processes ; this characteristic is related to the $H$ - and the $F$-independence ;

For $s_{f}$ referring only local variables or constants, the way the sequence $s_{f}$ is executable cannot change after the execution of $i_{0}$. More precisely, suppose that $i_{2}$ refers a variable $x$ and that $s_{f}$ is executable after $i_{0}$ for a value $x_{0}$ of $x$; as $x$ is local, the value of $x$ cannot change after $i_{0}$ is executed and before $s_{f}$ execution, and then the way that $s_{f}$ is executed does not change. So the $F$-Independence condition is fulfilled. As statements are executed by a process, $i_{0}$ cannot be re-executed before $s_{f}$ has been executed. The strongly $F$-Independence condition is then also fulfilled (in the Petri net model, it would mean that the transitions of the sequence do not access places also accessed by a transition modeling another process execution).

The same reasoning can be performed for $i_{0}$, but in this case would fulfill the $H$-independent and the strongly quasi-persistence conditions. As 
$i_{0}$ accesses only variables that cannot change when the process is not active, the statement $i_{0}$ can be delayed and then the $H$-independent and the strongly quasi-persistence conditions are fulfilled.

3. is the statement a guard (a first statement of a sequence in a branch of a selection)? in that case, the statement is potentially in competition with other statements in other branches of the selection structure (the process may have a choice); this characteristic is related to the quasi-persistent and the $H$-similar hypothesizes.

If there is no competition (the statement is not a guard) the $H$-similarity condition is fulfilled $(|I|=1)$. Others cases have to be discussed for each kind of statement.

The statement $i_{0}$ is followed by non blocking statements. The first case is when the sequence $s_{f}=$ atomic $\left\{i_{1} ; \ldots ; i_{k}\right\}$ is a non blocking sequence and $i_{1} ; \ldots ; i_{k}$ only refers local variables or constants (i.e. variables that are declared within the corresponding process or that are never assigned except at their declaration). In that case, a post-agglomeration of $i_{0}$ with the rest of the sequence can be performed. Indeed, as $s_{f}$ is non blocking, it can be executed as soon as $i_{0}$ has been executed and then the $F$-continuation condition is fulfilled. Now, as $i_{1} \ldots ; i_{k}$ refers only local variables or constants the way the sequences $s_{f}$ is executable cannot change after the execution of $i_{0}$. More precisely, suppose that $i_{2}$ refers a variable $x$ and that $s_{f}$ is executable after $i_{0}$ for a value $x_{0}$ of $x$; as $x$ is local, the value of $x$ cannot change before $s_{f}$ is executed, and then the way that $s_{f}$ is executed does not change when $i_{0}$ has been executed and $s_{f}$ not. So the $F$-Independence condition is fulfilled. As statements are executed by a process, $i_{0}$ cannot be re-executed before $s_{f}$ has been executed. The strongly $F$-Independence condition is then also fulfilled.

The statement $i_{\mathbf{0}}$ is not a guard. We suppose here that $i_{0}$ is not a guard and we examine three kinds of statements for $i_{0}$ : the blocking conditional statement $(\mathrm{x}==\mathrm{y})$ the assignment $(\mathrm{x}=\mathrm{y})$ and the receive operation on a channel $(\mathrm{q} ? \mathrm{x})$

1. Suppose first that $i_{0}$ is an assignment that does not refer to global variables (except constants). As $i_{0}$ accesses only variables that cannot change when the process is not active, the statement $i_{0}$ can be delayed and then the $H$ independent and the strongly quasi-persistence conditions are fulfilled. As the statement $i_{0}$ is not a loop, the divergence freeness is ensured. Moreover, as we agglomerate a single statement $\left(i_{0}\right)$ with a sequence $\left(s_{f}\right)$, the H-similarity condition is fulfilled $(|I|=1)$. Now, if $i_{1}$ is a blocking statement and if $i_{1}$ does not use variables modified by $i_{0}$ and does not modify variables accessed by $i_{0}$, we can safely replace the statement atomic $\left\{i_{0} ; i_{1} ; \ldots ; i_{k}\right\}$ by the statement atomic $\left\{i_{1} ; i_{0} ; \ldots ; i_{k}\right\}$. By this way we put the "blocking" statement at the beginning of the sequence which disables a possible interruption in the atomic statement execution.

2. Now, suppose that $i_{0}$ is a boolean expression and suppose that this expression does not refer to global variables (except constants). Then using the same reasoning, a pre-agglomeration can be performed on $i_{0}$ and $s_{f}$. 
3. When $i_{0}$ is a blocking reception on a channel we have to be careful. First, suppose that the channel is marked as "exclusive reader". This disables the possibility that a process takes a message that another process was waiting for (which will contradict the quasi-persistence condition). Then the $H$-independence condition implies that the reception of a message on the channel does not enable any action of an other process. In the general case this is not possible (a "reader" can unblock a "writer"). However, suppose that the user can mark a channel as "sufficient capacity" meaning that a writing statement on this channel will never be blocked; then, reading a message on such a channel cannot unblock a process waiting for writing. In such a case, a pre-agglomeration can be safely performed.

The statement $\boldsymbol{i}_{\mathbf{0}}$ is a guard. Now suppose that $i_{0}$ is the first statement of a selection structure (this applies also to a repetition statement)

if

$:: i_{0} ;$ atomic $\left\{i_{1} ; \ldots ; i_{k}\right\}$

$:: s_{1}$

$\because: \ldots$

$:: s_{n}$

$::$ else $s_{e}$

fi

where $s_{1}, \ldots, s_{n}$ and $s_{e}$ are sequences of actions (atomic or not).

1. First, suppose that $i_{0}$ is an assignment or a boolean expression that uses only local variables or constants. Suppose also that atomic $\left\{i_{1} ; \ldots ; i_{k}\right\}$ is a non blocking sequence, that each statement $s_{j}$ can be written $i_{0}^{j} . s_{j}^{\prime}$ with $s_{j}^{\prime}$ a non blocking sequence and that $i_{0}^{j}$ is an assignment or a boolean expression that uses only local variables or constants; then we can perform a pre-agglomeration of $i_{0}$ with the sequence atomic $\left\{i_{1} ; \ldots ; i_{k}\right\}$ simultaneously with a pre-agglomeration of each $i_{0}^{j}$ with the first statement of $s_{j}^{\prime}$. Indeed, the $H$-independence and the quasi-persistence are ensured due to the locality of variables used in statements. The $H$-similarity is obtained by the non blocking character of each sequence $s_{j}^{\prime}$ which ensures that if a given sequence $s_{j}^{\prime}$ is executable, then all other sequences $s_{j^{\prime}}^{\prime}$ are also executable.

2. Next, suppose that $i_{0}$ is a boolean expression using only local variables and constants. If all statements $s_{i}$ also begin with a boolean expression using only local variables and constants and if at most one of this boolean expression is true at a time, then $i_{0}$ can be pre-agglomerated with atomic $\left\{i_{1} ; \ldots ; i_{k}\right\}$. This is so because there is no really choice on the selection structure: at most one sequence is executable and the one which is executable does not change until it is executed.

3. The same reasoning can be applied when $i_{0}$ is a statement $q ? v_{0}\left(x_{0}\right)$ such that $q$ is a channel marked as an exclusive reader which does not block writers under the conditions that:

(a) each $s_{i}$ is also a statement $q$ ? $v_{i}\left(x_{i}\right)$, where $v_{0} \ldots v_{n}$ denotes different constant values, 
(b) $x_{0}, \ldots, x_{i}$ design local variables and

(c) there is no else part in the selection structure.

Indeed, in that case, there is no real choice (due to the different value of message type) and as there is no else part, the message reception can be delayed.

4. At last, suppose that all alternatives of a selection statement are atomic sequences; then, without modifying its behavior we can rewrite it into an atomic sequence that contains the selection statement as the unique statement.

The following algorithm (Algorithm 1) formalizes these Promela sources transformation rules.

\section{Experimentations}

We implemented these agglomerations in a tool (atomicSpin 12]). We applied our transformations on different models. Using the Spin tool (v. 4.3.0), we compute the total number of generated states when looking for all invalid end-states in the original and in the reduced model.

Consider the producer/consumer specification depicted on Figure 1. The resulting specification after agglomerations is depicted on Figure 2.

Consider process of type Reader: the sequence root?i; $j++$ can be transformed into atomic \{root?i; j++\} using the first rule (3.1.2) which corresponds to a post-agglomeration. Indeed, $j++$ is a non blocking statement using only local variable. Then, we can operate the transformation of the sequence $(j<=N)$; atomic $\{r \operatorname{root} ? i ; j++\}$ into the sequence atomic $\{(j<=N)$; root? $i ; j++\}$ using the second rules of subsection 3.1.4. Symmetric transformations can be applied in the code of the Writer process.

In the Table 1. we trace the number of reachable states for the original and the transformed model using the Spin partial order reduction in both cases (SPO

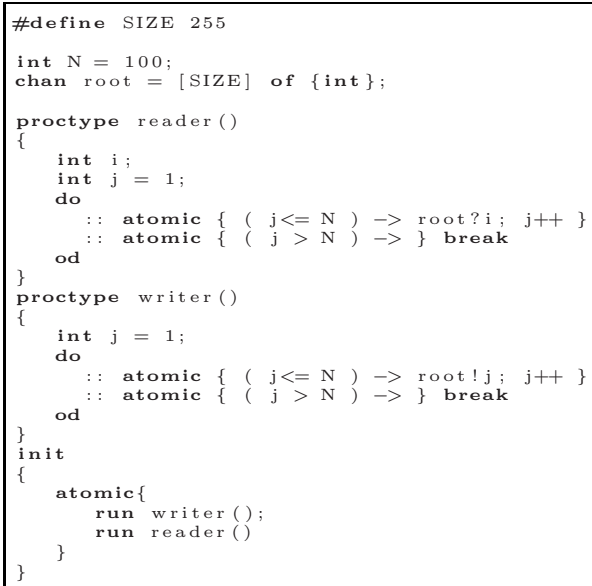

Fig. 2. The simple producer/consumer with automatically inferred atomic blocks 


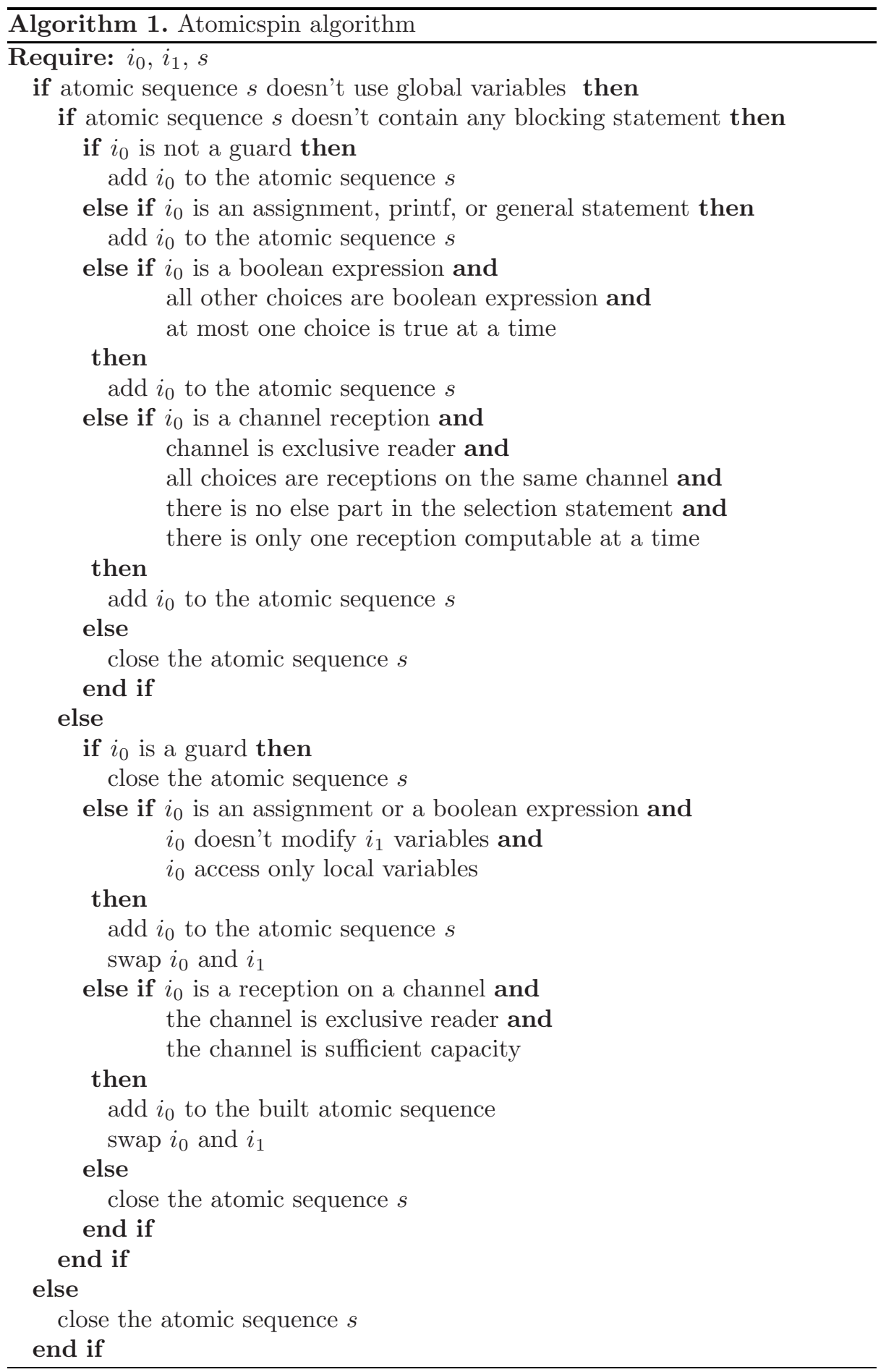


Table 1. Benchmarks

\begin{tabular}{|c|c|c|c|c|c|}
\hline \multirow[t]{2}{*}{ name } & \multirow[t]{2}{*}{ process } & \multicolumn{2}{|c|}{ states } & \multicolumn{2}{|c|}{ memory (Mo) } \\
\hline & & $\mathrm{SPO}$ & $\mathrm{APO}$ & $\mathrm{SPO}$ & $\mathrm{APO}$ \\
\hline \multirow[t]{5}{*}{ allocator } & 1 & 44 & 23 & 0 & 0 \\
\hline & 2 & 1818 & 646 & 0 & 0 \\
\hline & 3 & 42419 & 11876 & 6 & 2 \\
\hline & 4 & 637398 & 160242 & 107 & 26 \\
\hline & 5 & $7.77 \mathrm{e}+06$ & $1.89 \mathrm{e}+06$ & 1555 & 378 \\
\hline \multirow[t]{5}{*}{ leader } & 20 & 367 & 267 & 0 & 0 \\
\hline & 40 & 727 & 527 & 1 & 1 \\
\hline & 60 & 1087 & 787 & 2 & 2 \\
\hline & 80 & 1447 & 1047 & 4 & 3 \\
\hline & 100 & 1807 & 1307 & 7 & 5 \\
\hline \multirow[t]{5}{*}{ leader2 } & 2 & 84 & 60 & 0 & 0 \\
\hline & 3 & 356 & 254 & 0 & 0 \\
\hline & 4 & 2074 & 1482 & 0 & 0 \\
\hline & 5 & 14122 & 10082 & 3 & 2 \\
\hline & 6 & 106514 & 75986 & 29 & 21 \\
\hline \multirow{3}{*}{ petersonN } & 3 & 2999 & 2374 & 0 & 0 \\
\hline & 4 & 533083 & 383478 & 21 & 21 \\
\hline & 5 & - & - & - & - \\
\hline \multirow{5}{*}{$\overline{\text { philo1 }}$} & 2 & 42 & 20 & 0 & 0 \\
\hline & 4 & 1525 & 236 & 0 & 0 \\
\hline & 6 & 64944 & 3745 & 6 & 0 \\
\hline & 8 & $2.91 \mathrm{e}+06$ & 62712 & 373 & 8 \\
\hline & 10 & - & $1.02 \mathrm{e}+06$ & - & 155 \\
\hline \multirow[t]{5}{*}{ philo2 } & 10 & 189445 & 86407 & 20 & 9 \\
\hline & 11 & 706565 & 292125 & 84 & 35 \\
\hline & 12 & $2.61 \mathrm{e}+06$ & 955822 & 334 & 122 \\
\hline & 13 & $9.50 \mathrm{e}+06$ & $3.14 \mathrm{e}+06$ & 1293 & 427 \\
\hline & 14 & - & $1.02 \mathrm{e}+07$ & - & 1478 \\
\hline \multirow[t]{5}{*}{$\overline{\text { prod_cons }}$} & 200 & 161609 & 41009 & 172 & 44 \\
\hline & 400 & 558529 & 214789 & 596 & 229 \\
\hline & 600 & 967329 & 419589 & 1033 & 448 \\
\hline & 800 & $1.37 \mathrm{e}+06$ & 624389 & 1469 & 666 \\
\hline & 1000 & $1.78 \mathrm{e}+06$ & 829189 & 1906 & 885 \\
\hline \multirow[t]{5}{*}{ sort } & 50 & 5252 & 2705 & 7 & 3 \\
\hline & 100 & 20502 & 10404 & 51 & 26 \\
\hline & 150 & 45752 & 23104 & 172 & 87 \\
\hline & 200 & 81002 & 40804 & 406 & 204 \\
\hline & 250 & 126252 & 63304 & 791 & 398 \\
\hline
\end{tabular}


means Spin with partial order reductions, and APO means atomicSpin with the same partial order reductions). Agglomerations leads to a significant reduction of the state space size, with a quasi null cost in time (checking our conditions on Promela programs is a very simple task).

We also carried out experiments on classical examples of Promela models included in the standard distribution of Spin (the leader election, the distributed sort, the two versions of a cell-phone handoff strategy in a mobile network, the Peterson for N, the snooping cache) and some examples of our own (two version of the well known dining philosophers and a task allocator modeling a clientserver application).

Agglomerations improve the state space reduction in all cases. Except for the leader election, the reduction factor is at least 2 even when partial-order reductions are enabled and agglomerations performed on the dining philosophers model achieved reductions of the state space by a factor 20 .

\section{Related Works on Syntactical Model Reductions}

First works concerning reduction of sequences into atomic actions for simplification purpose was performed by Lipton in [13. Lipton focused only on deadlock property preservation. Using parallel program notations of Dijkstra he defined "left" and "right" movers. Roughly speaking, a "left" (resp. "right") mover is a local process statement that can be moved forward (resp. delayed) w.r.t. statements of others processes without modifying the halting property. Lipton then demonstrated that, in principle, the statement $P(S)$, where $S$ is a semaphore, is a "left" mover and V(s) is a "right" mover. Then Lipton proved that some parallel program are deadlock free by moving $P(S)$ and $V(S)$ statements and by suppressing atomic statements that have no effect on variables. However, two difficulties arise: the reduction preserves only deadlocks and the application conditions are difficult to be checked.

Cohen and Lamport propose in [14] assumptions on TLA specifications under which they define a reduction theorem preserving liveness and safety properties. This work fixes the reduction theorem in a "high" level formalism which can be a clear advantage for defining specific utilization. However, it's also its main drawback since it is based on the hypothesis that some actions commute, but no effective way is proposed to check whether this assumption holds.

More recently, Cohen, Stoller, Qadeer, and Flanagan [15], [16], 17] leveraged Lipton's theory of reduction to detect transactions in multi-threaded programs (and consider these transactions as atomic actions in the model checking step). Stoller and Cohen propose in [15] a reduction theorem based on omega algebra that can be applied to models of concurrent systems using mutual exclusion for access to selected variables. However, they use a restricted notion of "left" mover and a better reduction ratio can be obtained by applying more accurate reductions (as demonstrated in [18). Moreover, their reductions are justified by the correct use of "exclusive access predicates" and by the respect of a specific synchronization discipline. These predicates may be difficult to compute and no effective algorithm is given to test that the synchronization discipline is respected. 
Flanagan and Qadeer noted in [17] that the previous authors use only the notion of "left" mover and proposed an algorithm that uses both "left" and "right" mover notions to infer transactions. However, this algorithm is based on access predicates that can be automatically inferred only for specific programs using lock-based synchronization. Moreover, as they use both "left" and "right" movers to obtain a better reduction ratio and as they do not fix sufficient restrictive application conditions, their reduction theorem do not preserve deadlock.

In Petri nets formalism, the first works concerning reductions have been performed by Berthelot [19. The link between transition agglomerations (the most effective structural reductions proposed by Berthelot) and general properties, expressed in LTL formalism, is done in 20.

We proposed in [6] new Petri nets reductions that cover a large range of patterns by introducing algebraic conditions whereas the previously defined ones rely solely on structural conditions. We adapted them in [7] to colored Petri nets which are an abbreviation of Petri nets and define a concise formalism for the modeling of concurrent software. We showed here that these reductions can also be adapted to Promela specifications leading to simple syntactical rules which permit a significant reduction of the combinatory while preserving properties of the model.

\section{Conclusion}

We demonstrate in this paper that efficient Petri nets reductions can be used to significantly reduce the state space size of a Promela specification. We propose simple syntactical rules allowing the automatic building of atomic sequences. Our experiments highlight the efficiency of these approaches. A first implementation of these rules has already been developed [12. Our experience with Petri nets allows us to expect even better reductions for more complex models. Based on these experimentations we plan to adapt these transformation rules to automatically infer transactions in concurrent software written in Ada or Java in the near future.

\section{References}

1. Wolper, P., Godefroid, P.: Partial-order methods for temporal verification. In: Best, E. (ed.) CONCUR 1993. LNCS, vol. 715, pp. 233-246. Springer, Heidelberg (1993)

2. Godefroid, P., Wolper, P.: Using partial orders for the efficient verification of deadlock freedom and safety properties. Form. Methods Syst. Des. 2(2), 149-164 (1993)

3. Valmari, A.: On-the-fly verification with stubborn sets. In: Courcoubetis, C. (ed.) CAV 1993. LNCS, vol. 697, pp. 397-408. Springer, Heidelberg (1993)

4. Emerson, A., Prasad Sistl, A.: Symmetry and model checking. In: Courcoubetis, C. (ed.) CAV 1993. LNCS, vol. 697, pp. 463-478. Springer, Heidelberg (1993)

5. Sistla, A.P.: Symmetry reductions in model-checking. In: Zuck, L.D., Attie, P.C., Cortesi, A., Mukhopadhyay, S. (eds.) VMCAI 2003. LNCS, vol. 2575. Springer, Heidelberg (2002)

6. Haddad, S., Pradat-Peyre, J.: Efficient reductions for LTL formulae verification. Technical report, CEDRIC, CNAM, Paris (2004) 
7. Evangelista, S., Haddad, S., Pradat-Peyre, J.F.: New coloured reductions for software validation. In: Workshop on Discrete Event Systems (2004)

8. Haddad, S., Pradat-Peyre, J.-F.: New efficient petri nets reductions for parallel programs verification. Parallel Processing Letters 16(1), 101-116 (2006)

9. Evangelista, S., Kaiser, C., Pradat-Peyre, J.F., Rousseau, P.: Quasar: a new tool for analysing concurrent programs. In: Rosen, J.-P., Strohmeier, A. (eds.) Ada-Europe 2003. LNCS, vol. 2655. Springer, Heidelberg (2003)

10. Holzmann, G.J.: The model checker SPIN. Software Engineering 23(5), 279-295 (1997)

11. Pajault, C., Pradat-Peyre, J.: Static reductions for promela specifications. Technical Report 1005, Conservatoire National des Arts et Métiers, laboratoire Cedric, Paris, France (2006)

12. http://quasar.cnam.fr/atomicSpin/

13. Lipton, R.J.: Reduction: a method of proving properties of parallel programs. Commun. ACM 18(12), 717-721 (1975)

14. Cohen, E., Lamport, L.: Reduction in TLA. In: International Conference on Concurrency Theory, pp. 317-331 (1998)

15. Stoller, S.D., Cohen, E.: Optimistic synchronization-based state-space reduction. In: Garavel, H., Hatcliff, J. (eds.) ETAPS 2003 and TACAS 2003. LNCS, vol. 2619. Springer, Heidelberg (2003)

16. Flanagan, C., Qadeer, S.: A type and effect system for atomicity. In: Proceedings of the ACM SIGPLAN 2003 conference on Programming language design and implementation, pp. 338-349. ACM Press, New York (2003)

17. Flanagan, C., Qadeer, S.: Transactions for software model checking. In: Cook, B., Stoller, S., Visser, W. (eds.) Electronic Notes in Theoretical Computer Science, vol. 89. Elsevier, Amsterdam (2003)

18. Evangelista, S., Haddad, S., Pradat-Peyre, J.: Coloured Petri nets reductions for concurrent software validation. Technical report, CEDRIC, CNAM, Paris (2004)

19. Berthelot, G.: Checking properties of nets using transformations. In: Rozenberg, G. (ed.) Advances in Petri nets. LNCS, vol. 222. Springer, Heidelberg (1985)

20. Poitrenaud, D., Pradat-Peyre, J.: Pre and post-agglomerations for LTL model checking. In: Nielsen, M., Simpson, D. (eds.) ICATPN 2000. LNCS, vol. 1825. Springer, Heidelberg (2000)

\section{A Agglomerated Petri Net Definition}

Proposition 1. The incidence matrices $W, W^{-}$and $W^{+}$can be extended to matrices indexed by $P \times T^{*}$ by the following recursive definition:

$-W(p, \lambda)=W^{-}(p, \lambda)=W^{+}(p, \lambda)=0$ and $W\left(p, s_{1} . t\right)=W\left(p, s_{1}\right)+W(p, t)$

$-W^{-}\left(p, s_{1} . t\right)=\operatorname{Max}\left(W^{-}\left(p, s_{1}\right), W^{-}(p, t)-W\left(p, s_{1}\right)\right)$

$-W^{+}\left(p, s_{1} \cdot t\right)=W(p, s)+W^{-}(p, s)$

such that this extension is compatible with the firing rule, i.e.

$\forall s \in T^{*}, m[s\rangle m^{\prime} \Longleftrightarrow \forall p \in P, m(p) \geq W^{-}(p, s)$ and $m^{\prime}(p)=m(p)+W(p, s)$

Definition 8 (Reduced net). The reduced Petri net $\left(N_{r}, m_{0}\right)$ is defined by:

- $P_{r}=P, T_{r}=T_{0} \cup_{i \in I}\left(H_{i} \times F_{i}\right)$ (we note $h f$ the transition $(h, f)$ of $\left.H_{i} \times F_{i}\right)$;

$-\forall t_{r} \in T_{0}, \forall p \in P_{r}, W_{r}^{-}(p, t)=W^{-}(p, t)$ and $W_{r}^{+}(p, t)=W^{+}(p, t)$

$-\forall i \in I, \forall h f \in H_{i} \times F_{i}, \forall p \in P_{r} W_{r}^{-}(p, h f)=W^{-}(p, h . f)$ and $W_{r}^{+}(p, h f)=$ $W^{+}(p, h . f)$ 